Research in Astron. Astrophys. 2011 Vol. 11 No. XX, 000-000

http://www.raa-journal.org http://www.iop.org/journals/raa

$\boldsymbol{R}$ esearch in

Astronomy and

Astrophysics

Received [year] [month] [day]; accepted [year] [month] [day]

\title{
On the origin of $\mathrm{X}$-shaped radio galaxies
}

\author{
Gopal-Krishna $^{1}$, Peter L. Biermann ${ }^{2,3,4,5,6}$, László Á. Gergely ${ }^{7,8}$ and Paul J. Wiita ${ }^{9}$ \\ 1 National Centre for Radio Astrophysics, TIFR, Pune University Campus, Post Bag 3, Ganeshkhind, \\ Pune 411007, India; krishna@ncra.tifr.res.in \\ 2 Max Planck Institute for Radioastronomy, Auf dem Hügel 69, 53121 Bonn, Germany; \\ plbiermann@mpifr-bonn.mpg.de \\ 3 Department of Physics \& Astronomy, University of Bonn, Germany \\ ${ }^{4}$ Department of Physics \& Astronomy, University of Alabama, Tuscaloosa, AL, USA \\ 5 Department of Physics, University of Alabama at Huntsville, AL, USA \\ ${ }^{6}$ FZ Karlsruhe, and Physics Department, University of Karlsruhe, Germany \\ 7 Department of Theoretical Physics, University of Szeged, Tisza Lajos körút 84-86, 6720 Szeged, \\ Hungary; gergely@physx.u-szeged.hu \\ 8 Department of Experimental Physics, University of Szeged, Dóm tér 9, 6720 Szeged, Hungary \\ ${ }^{9}$ Department of Physics, The College of New Jersey, P.O. Box 7718, Ewing, NJ 08628-0718, USA; \\ wiitap@tcnj.edu
}

\begin{abstract}
After a brief, critical review of the leading explanations proposed for the small but important subset of radio galaxies showing an X-shaped morphology (XRGs) we propose a generalized model, based on the jet-shell interaction and spin-flip hypotheses. The most popular scenarios for this intriguing phenomenon invoke either hydrodynamical backflows and over-pressured cocoons or rapid jet reorientations, presumably from the spin-flips of central engines following the mergers of pairs of galaxies, each of which contains a supermassive black hole (SMBH). We confront these models with a number of key observations and thus argue that none of the models is capable of explaining the entire range of salient observational properties of XRGs, although some of the arguments raised in the literature against the spin-flip scenario are probably not tenable. We then propose here a new scenario which also involves galactic mergers but would allow the spin of the central engine to maintain its direction. Motivated by the detailed multi-band observations of the nearest radio galaxy, Centaurus A, this new model emphasizes the role of interactions between the jets and the shells of stars and gas that form and rotate around the merged galaxy and can cause temporary deflections of the jets, occasionally giving rise to an X-shaped radio structure. Although each of the models is likely to be relevant to a subset of XRGs, the bulk of the evidence indicates that most of them are best explained by the jet-shell interaction or spin-flip hypotheses.
\end{abstract}

Key words: galaxies - active; galaxies - jets; gravitational radiation; interstellar medium; radio sources - continuum

\section{INTRODUCTION}

Morphologies of powerful extragalactic radio sources on kiloparsec and larger scales can typically be related to a single pair of anti-parallel jets of relativistic plasma, ejected from a "central engine" located 
at the active nucleus of a massive early-type galaxy (e.g., Begelman, Blandford \& Rees 1984). In some rare cases, an additional inner pair (or pairs) of radio lobes is observed, roughly aligned with the outer, presumably older, lobe pair. These, so called, "double-double" radio galaxies are believed to be examples of restarted nuclear activity (Lara et al. 1999; Schoenmakers et al. 2000; Saripalli, Subrahmanyan \& Udaya Shankar 2002; Konar et al. 2006), in which the new jet pair broadly retains its alignment with the older jets. In one scenario, the interruption in the jet activity is attributed to the disruption of the accretion disk due to the inspiral of a second supermassive black hole through the disk surrounding the larger one (Liu et al. 2003). In the last few years an even more striking morphological class of radio galaxies has drawn increasing attention; they exhibit two pairs of radio lobes associated with a single parent galaxy, such that their axes are grossly misaligned. Although the existence of such "winged" or "X-shaped" radio galaxies (XRGs) has been known for the past few decades (e.g., Högbom \& Carlsson 1974; Ekers et al. 1978; Leahy \& Williams 1984; Leahy \& Parma 1992), the recent upsurge of interest in XRGs stems from the distinct possibility that their precursors were potential sites of the intense gravitational radiation which is expected in a merger of the two supermassive black holes (SMBHs) associated with the merged galaxy pair. Another simultaneous outcome expected from such a merger is a sudden change in the spin axis of the $\mathrm{SMBH}$, which can find manifestation in the emergence of a new jet pair at an axis grossly misaligned from the axis of the jets prior to the merger, producing the so called, "spin-flip" model for XRGs (e.g., Rottmann 2001; Zier \& Biermann 2001, 2002; Merritt \& Ekers 2002; Biermann et al. 2005; Gergely \& Biermann 2009, hereafter GB09). An earlier explanation, specifically devised for the rare dumbbell galaxies (elliptical galaxies with two nuclei, such as the XRG NGC 326), posited a more gradual reorientation of the large-scale jets during a close passage between two elliptical galaxies (Wirth, Smarr \& Gallagher 1982).

The explanation invoking a SMBH merger accords well with the extensively discussed model of powerful double radio sources that argues that jet formation is often triggered by a galaxy merger, particularly the merger of a gas-rich galaxy with a large elliptical (e.g., Begelman et al. 1984; Heckman et al. 1986; Blandford 1992; Wilson \& Colbert 1995; Wang \& Biermann 1998). The possibility that inversion symmetric radio galaxy morphologies were due to a SMBH binary in a single galactic nucleus was first suggested by Begelman et al.1980). Significant additional support for this scenario comes from the growing evidence in favor of the presence of binary black hole systems within the nuclei of active galaxies. One line of evidence comes from recent discoveries of double-peaked broad low-ionization lines separated by $>2000 \mathrm{~km} \mathrm{~s}^{-1}$ (Komossa, Zhou \& Lu 2008; Boroson \& Lauer 2009; Shields, et al. 2009); however, it must be noted that these systems could just be superpositions of two active galactic nuclei (AGN) within the same cluster of galaxies (e.g., Dotti \& Ruszkowski 2009). X-ray imaging and spectroscopy indicate that both the optical nuclei (separated by a projected distance of $\sim 1.3 \mathrm{kpc}$ ) of the ultraluminous infrared galaxy NGC 6240 are active and thus house SMBHs (Komossa et al. 2003). It has been noted that the radio emission from NGC 1275 is consistent with accelerating jet precession and this could imply the presence of a binary SMBH system with a rapidly changing orbital separation (Liu \& Chen 2007). More interestingly, double-peaked low-ionization emission lines in the nucleus of a galaxy hosting an XRG J1130+0058 have been reported (Zhang, Dultzin-Hacyan \& Wang 2007). Very recently, the detection of a galaxy containing three active nuclei, SDSS J1027+1749, has been reported (Liu et al. 2011); here the secondary emission line nuclei are offset by 2.4 and $3.0 \mathrm{kpc}$ in projected separation and by 450 and $111 \mathrm{~km} \mathrm{~s}^{-1}$ in velocity. Further evidence for binary SMBHs comes from models of the periodic intensity variations of some blazars, notably OJ 287 (Sillanpää et al. 1988; Valtonen et al. 2008). The detection in the RG $0402+379$ of two compact, VLBI sources of variable radio emission, separated by only $7.3 \mathrm{pc}$, also presents a strong case for a SMBH merger in progress (Rodriguez et al. 2006). An earlier stage leading to an eventual merger is very possibly exemplified by the highly disturbed radio morphology in the compact steep radio spectrum quasar, FIRST J164311.3+315618, which is in a binary system with a projected separation of $15 \mathrm{kpc}$ (Kunert-Bajraszewska \& Janiuk 2011).

The slow accumulation of observational evidence notwithstanding, the occurrence of SMBH mergers has been long firmly believed on the basis of simple theoretical arguments. For instance, the PressSchechter model (Press \& Schechter 1974) is premised on an ongoing merger process for galaxy formation and this yields a good approximation to the galaxy luminosity function. When this model (or more 
sophisticated cosmological simulations) is coupled with the more recent paradigm that a very large fraction of galaxies undergo central activity emitting a compact radio signature (e.g. Perez-Fournon \& Biermann 1984; Nagar et al. 2001; Nagar, Falcke \& Wilson 2005; Capetti et al. 2010) and that most galaxies harbor a central BH (e.g., Kormendy \& Richstone 1995; Faber et al. 1997; Magorrian et al. 1998), the expectation is that SMBH mergers are not uncommon.

XRGs are now known to constitute a significant fraction (up to 10\%) of the more powerful, edgebrightened, FR II (Fanaroff \& Riley 1974) radio galaxies in the 3CRR catalog (see, Leahy \& Williams 1984, hereafter LW84; Leahy \& Parma 1992). Many new XRGs, or at least good XRG candidates, have recently been found and characterized (e.g., Cheung 2007, hereafter C07; Cheung et al. 2009; Saripalli \& Subrahmanyan 2009, hereafter SS09), mostly using the FIRST radio survey (Becker, White \& Helfand 1995). Usually XRGs are found to have radio powers near to the radio luminosity of the Fanaroff \& Riley (1974) division between FR I and FR II sources at $P_{178 \mathrm{MHz}} \approx 2 \times 10^{25} \mathrm{~W} \mathrm{~Hz}^{-1} \mathrm{sr}^{-1}$ (Landt, Cheung \& Healey 2010 and references therein). Therefore it is striking that not a single case is found where both lobe pairs are of FR II type; usually only one (but sometimes neither) of the two lobe pairs shows radio hot spots near the lobes' extremities, while the other lobe pair lacks compact features altogether and normally is distinctly less collimated (e.g., LW84; SS09). That pair is therefore termed as "secondary lobes", or "wings", in contrast to the other lobe pair, called "primary lobes".

Several authors have argued that the wings arise due to diversion of the backflow of synchrotron plasma in the two primary lobes. The diversion could be preferrentially into the cavities left over by the lobes of a previously active phase (LW84), or due to buoyancy pressure driven by a steep pressure gradient in the ambient medium (e.g., Worrall, Birkinshaw \& Cameron 1995; Kraft et al. 2005; Miller \& Brandt 2009; SS09; Hodges-Kluck et al. 2010b). Another variant to this buoyant backflow model is the "over-pressured cocoon" model where a rapid build-up of the pressure within the radio cocoon leads to a collimated supersonic outflows of the lobe's synchrotron plasma as it squirts out along the directions of the fastest declining ambient pressure (Capetti et al. 2002, hereafter C02).

In a less discussed variant of this class of models (Gopal-Krishna, Biermann \& Wiita 2003, hereafter GBW03), the backflow gets diverted as it impinges, from opposite sides, on to an inclined 'superdisk' of thermal plasma around the host elliptical. The existence of such superdisk structures, with mean thickness of $\approx 30 \mathrm{kpc}$, around a number of FR II RGs has been inferred from the sharp strip-like emission gaps seen between their radio lobe pairs (Gopal-Krishna \& Wiita 2000a, 2009). There are a number of physically reasonable models for the formation of the superdisks (e.g., Gopal-Krishna, Wiita \& Joshi 2007; Gopal-Krishna \& Wiita 2009). One model worth highlighting in the present context considers the physics of the final stage of the spin-flip of merging BHs, which is marked by a rapid precession of the $\mathrm{BH}$ axis due to which the jets swing about rapidly (GB09). These swinging jets can produce a narrowing cone-like wind through fast and massive entrainment of the surrounding gas, thus potentially giving rise to a superdisk (GB09). If true, this picture could be useful in identifying the best candidates for an imminent merger of SMBHs.

In Section 2 we contrast the different explanations proposed for the XRG phenomenon with the current observational results. In Section 3 we propose a new additional mechanism enriching the phenomenology of galaxy-galaxy interactions when one or both of the galaxies harbor a central supermassive black hole: the jet-shell interaction model for XRGs. Section 4 summarizes our main conclusions.

\section{MODELS FOR XRGS}

In this section we briefly recapitulate the three main explanations, including some of their variants, that have been proposed for the XRG phenomenon.

First, however, we summarize some key results concerning the orientations of radio emission axes and optical shells with respect to the optical shape of their host galaxies. For many years, only rather small samples of RGs $(\sim 100)$ could be used and, while there were claims of a correlation between radio emission and the minor axes of the host elliptical galaxies (e.g., Palimaka et al. 1979), no significant correlation was found in some other studies (e.g., Sansom et al. 1987). 
An important result has come from the recent work of Battye \& Browne (2009) who employed a much larger sample of $>14,000$ galaxies obtained from matching the FIRST radio survey with the SDSS optical catalog. They have demonstrated that late type galaxies bear an expected strong correlation between the optical major axis and the radio emission axis, presumably arising from the association of the radio emission with star formation in their disks. On the other hand, for early type galaxies they do find a significant correlation between the radio axis and the optical minor axis; however, this correlation is dominated by the galaxies with lower ratios of radio to optical powers. For stronger radioloud ellipticals, with which we are concerned here, no significant correlation of radio axis with optical axis was found (Battye \& Browne 2009).

At the same time, as shown in Capetti et al. (2002), XRGs are only hosted by substantially elongated ellipticals (ellipticity, $\eta \gtrsim 0.2$ ). In addition, $\mathrm{C} 02$ found that the principal radio axis defined by the two hot spots of the primary lobe pair shows a distinct tendency to be close to the optical major axis of the parent elliptical. Perhaps an even tighter alignment exists between the host galaxy's minor axis and the secondary radio axis as defined by the pair of the wings (C02). Both of these trends have been confirmed recently by SS09 and H-K10a using larger datasets. The latter authors inferred the minor axis from the distribution of hot, X-ray emitting gas detected with Chandra around the ellipticals in their sample. Hence for the XRG subset of stronger RGs, all studies agree that the primary radio lobe pair is preferentially oriented toward the major optical axis of the host elliptical (C02; SS09; HodgesKluck et al. 2010a, hereafter H-K10a), though the samples on which this conclusion is based are still comparatively small.

In certain cases a Z-symmetric morphology of the secondary lobes was observed (i.e, a lateral offset between the ridge lines of the two secondary lobes; see, Gopal-Krishna, Biermann \& Wiita 2003). We later show how such sources can provide a powerful discriminant between different XRG models.

Another more general important result, but one we believe could be particularly relevant for XRGs, concerns optical shells around elliptical galaxies. These optical shells, which probably arise during the course of a merger of an elliptical with a disky galaxy (e.g., Quinn 1984), are preferentially located along the major axes of post-merger ellipticals (e.g., Malin \& Carter 1983; Sect. 3).

Important evidence in favor of mergers playing an important role in XRGs comes from a recent study of 29 XRGs which were recently compared to a control sample of 36 RGs with "normal" morphologies but similar redshifts and optical luminosities (Mezcua et al. 2010). These authors find that the members of the XRG sample have, on average, significantly more massive SMBH, as would be expected if a merger engendered the change in jet direction in XRGs. In addition, more direct evidence for merger has recently come from the recent detection of shells in two XRGs, 3C 403 (Almeida et al. 2011) and 4C+00.58 (Hodges-Kluck et al. 2010b).

\section{1 “Twin-AGN" Model for XRGs}

This model is inspired by the existence of elliptical galaxies with double nuclei, e.g., NGC 326 (Battistini et al. 1980; Worrall et al. 1995). In this model the pair of twin radio lobes is considered to be two independent radio doubles associated with a close pair of active SMBHs inside a merging pair of massive ellipticals (Lal \& Rao 2007). This model appears particularly appealing in case the dynamical friction is insufficient, thereby stalling the approach of the two SMBHs. However, such stalling is normally not expected (e.g., Zier 2007; Sesana, Hardt \& Madau 2007; Hayasaki 2009; GB09, and references therein), as also inferred from extensive VLBI imaging observations (Burke-Spolaor 2011). In addition, this situation has a very low probability, in that it requires both central engines to be simultaneously launching jets.

While this "twin AGN" picture has the advantage of conceptual simplicity, it fails to explain why none of the XRGs has both its lobe pairs of the FR II type. Nor does it explain the preference of the primary lobe to be oriented towards the major axis of the optical host galaxy (C02; SS09; H-K10a). Furthermore, it is particularly hard in this picture to understand the often observed Z-symmetric morphology of the secondary lobes (Gopal-Krishna, Biermann \& Wiita 2003). On all these grounds, it is 
evident that while this model might account for a tiny fraction of XRGs, it is untenable for the bulk of the XRG population.

\subsection{Back-flow Diversion Models}

In the backflow diversion model for the formation of secondary lobe pair, (e.g., LW84, Worrall et al. 1995; Kraft et al. 2005), the (denser) ISM of the host elliptical galaxy plays a key role by exerting buoyancy pressure on the backflowing synchrotron plasma within the two primary lobes. As a result, the synchrotron plasma is diverted away from the lobe axis, along the direction of the fastest declining ISM pressure. In this way, the strong tendency for the wings to align with the optical minor axis of the host elliptical can be readily understood. Although appealing for its simplicity, as noted by several authors (e.g., Dennett-Thorpe et al. 2002), this hydrodynamic backflow model is severely challenged by the key observation that in several XRGs the wings are found to be distinctly more extended than the primary lobes, even though the latter are supposed to be advancing supersonically with respect to the external medium (whose ram pressure causes a bright hot spot marking the leading edge of the lobe), in contrast to the subsonically growing wings. Examples of such XRGs can be found, e.g., in Leahy \& Parma (1992); SS09 and Sect. 4.

To circumvent this problem, $\mathrm{C} 02$ proposed that the wings can form due to lateral expansion of the over-pressured cocoon of the double radio source, along the steepest gradient in the ISM pressure (i.e., roughly along the host galaxy's minor axis, assuming that the galaxy has high ellipticity, making its ISM asymmetric). The lateral expansion of the synchrotron plasma could well occur in the form of a pair of loosely collimated supersonic flows of the synchrotron plasma out of the over-pressured cocoon, via a "de Laval nozzle" formation (e.g., Blandford \& Rees 19741) This mechanism for the formation of secondary lobes can possibly allow them to grow even longer than the primary lobes, as argued by $\mathrm{C} 02$ based on two-dimensional hydrodynamical simulations. As these simulated "outflows" may be enhanced by the imposed cylindrical symmetry, the result needed to be verified by the use of threedimensional (3D) simulations. Very recently, such 3D simulations have been performed and they showed that under rather special circumstances such outflows with secondary lobes with lengths comparable to those of the primary lobes could indeed occur (Hodges-Kluck \& Reynolds 2011). Furthermore, given the appropriate viewing angle, such secondary lobes could appear to have a larger projected length than the primary ones (Hodges-Kluck \& Reynolds 2011; Wiita, Sobczak \& Starr 2011).

Secondly, in this scenario, a large misalignment between the primary and secondary lobe pairs (a prerequisite for an XRG classification) would be realizable only in those cases where the jets happen to be ejected roughly along the optical major axis of the parent elliptical galaxy. This selection bias could possibly force the backflow diversion model to be consistent with the observed tendency for the primary lobes in XRGs to be aligned with the optical major axis of the host galaxy, for if the jets emerged roughly along the host's minor axis to begin with then any cocoon outflows would tend to coincide with the original jet direction itself and the source would not be classified as an XRG at all.

The main morphological features of XRGs can be reproduced satisfactorily in this model. Nonetheless, it is evident that such a scenario can only work provided the jets are not very powerful, and therefore unable to exit quickly from the denser central region of the ISM, and possibly also from the intra-cluster medium (according to H-K10a), before a substantial cocoon has developed. Indeed, numerical simulations of more powerful jets crossing interstellar/intergalactic medium boundaries show only modest sideways flows from the backflowing plasma and thus only "stubby" wings (e.g., Hooda \& Wiita 1996, 1998). Still, the over-pressured cocoon model does explain why radio wings do not form in general and, more specifically, why they do not usually form in the weaker, edge-dimmed, FR I sources: such RGs simply do not generate the backflow needed to form over-pressured cocoons. Note that recently SS09 have highlighted cases of a few XRGs where even the better collimated (primary) lobe pair appears to have the FR I morphology. They suggest that probably these lobes too had an FR II past

\footnotetext{
1 We note that this physical concept of a spherical explosion in a stratified atmosphere leading to a jet-like formation of flow goes back to Kompaneets [1960] and was discussed in detail by Zeldovich \& Raizer [1966].
} 
when their hotspots generated strong backflows. Such a morphological transformation at later evolutionary stages has been long predicted from dynamical considerations (e.g., Gopal-Krishna \& Wiita 1988, 2000b; Gopal-Krishna 1991; Falle 1991; Bicknell 1994, 1995; Kaiser \& Alexander 1997; Nakamura et al. 2008).

The concept of an over-pressured cocoon finds some support from a recent study of the optical spectra of 53 XRGs, by Landt, Cheung \& Healey (2010) who find that unusually hot plasma (T > $15,000 \mathrm{~K}$ ) commonly exists in the nuclear regions of XRGs. Still, it remains unclear if the over-pressure extends all the way to their cocoons. The difficulty is further underscored by the case of the XRG NGC 326 in which the wings are not only a few times longer than the primary lobes but the "foot" of the eastern wing is located about $50 \mathrm{kpc}$ from the nucleus, i.e., well outside the ISM of the galaxy (e.g., Murgia et al. 2001; Worrall et al., 1995). This situation makes it hard to identify a mechanism to ensure confinement of the hypothesized over-pressured cocoon.

Finally, we note that in support of the backflow origin of XRG wings (vis-à-vis the spin-flip scenario described in Section 2.3), SS09 have highlighted a few cases where an inner pair of radio lobes is seen fairly well aligned with the primary lobe pair, indicating that nuclear activity in those sources has restarted practically along the same axis. In Section 2.3 we shall propose alternative interpretations for this result.

\subsection{Rapid Jet Reorientation Models}

In this radically different scheme, the wings are envisioned to be relic emission from the radio lobes created during an earlier phase of nuclear activity when the jets were oriented in that direction. The jet orientation then underwent a rapid change, possibly an abrupt "flip", resulting in the currently fed primary lobe pair. Models proposed for the jet reorientation invoke either precession or other realigning mechanisms (Ekers et al. 1978; Rees 1978; Klein et al. 1995; Dennett-Thorpe et al. 2002; FalcetaGonçalves et al. 2010; Hodges-Kluck et al. 2010b). A particularly detailed model argues that the central SMBH is realigned with the binary orbital plane during a process that involves a strong interaction between the binary and the accretion disk that twists and warps the disk which quickly realigns the spin axis of the SMBH ejecting the jets (Liu 2004). Alternatively, the jet realignment can be a more violent process involving the coalescence of the jetted SMBH with another SMBH in the throes of a galaxy merger (Rottmann 2001; Zier \& Biermann 2001; Merritt \& Ekers 2002; Dennett-Thorpe et al. 2002; GBW03). This latter mechanism is particularly interesting since, it also results in a copious emission of gravitational waves, which for the lower mass range of SMBHs, lies in the Laser Interferometer Space Antenna (LISA) frequency band.

In an earlier paper (GBW03) we argued how the spin-flip scenario can also readily account for the Z-symmetry about the nucleus, displayed by many of the wing-pairs. Pointing out the Z-distortion, GBW03 showed how it can arise prior to the spin-flip, as the jets propagated through the ISM of the massive elliptical host. The outer portion of the ISM already had been set in slow rotation by the captured galaxy (roughly along its original orbital plane) as it spiraled into the radio-loud elliptical, eventually culminating in the coalescence of their black holes (see, also, Noel-Storr et al. 2003; Heinz et al. 2008). Thus, at large distances from the galactic nucleus the two jets, having been slowed down, would be diverted by the rotating ISM in opposite directions. The deflected flows can exist for extended periods and can arise at substantial distances from the source galaxy. Note that a recent study of a Z-shaped FR I RG, NGC 3801, provides strong evidence for the presence of a recent merger and a large fast rotating gas disk interacting with the jets (Hota et al. 2009).

While the large misalignment between the two lobe pairs is statistically expected in the spin-flip scenario, it might seem difficult to understand the observed strong tendency for the primary lobes to align with the optical major axis of the host elliptical (C02; SS09; H-K10a). However, if the wings are forced into alignment with the optical minor axis due to the buoyancy pressure of the ISM of the parent galaxy acting on the backflowing synchrotron plasma, the trend for the primary lobes to be broadly aligned with the optical main axis (Capetti et al. 2002; SS09) could follow simply from the requirement 
of a large spin-flip angle, or else the source would often end up being classified as a double-double RG, as e.g., in the case of J1453+3309 (Konar et al. 2006).

Evidence consistent with significant spin-flips comes from RGs that appear to have restarted and have inner and outer structures that are significantly misaligned. For example, $1448+63$ shows a nearly $90^{\circ}$ misalignment between its pc and kpc structures (Giovannini et al. 2005), although such differences can be exaggerated if the jet is relativistic and pointing close to the line-of-sight. Less severe misalignments, but on much larger scales, and hence not likely to be affected by such projection effects, are present in other sources. Examples include the giant radio galaxy J0116- 473 (Saripalli, Subrahmanyan \& Udaya Shankar 2002), J1453+3309 (Konar et al. 2006) and 3C 293 (Evans et al. 1999).

In several XRGs an inner compact pair of radio lobes is seen and is roughly aligned with the primary lobe axis (see, SS09); this double-double morphology of the primary lobes, can be easily reconciled with the spin-flip model and the usual expectation that double-double RGs involve restarted jets (e.g. Lara et al. 1999). Note that in a few of the double-double XRGs, highlighted in SS09, the outer lobes lack hot spots and these have been interpreted as the lobes which had an FR II morphology previously (SS09). The possibility of large-scale jets/lobes transforming from FR II to FR I at later stage in their lives has also been noted in earlier studies (Gopal-Krishna \& Wiita 1988; Gopal-Krishna 1991; Falle 1991; Bicknell 1994; Kaiser \& Alexander 1997; Nakamura et al. 2008; Kawakatu et al. 2009).

A modified version of this scheme was proposed by Dennett-Thorpe et al. (2002), based on their analysis of radio spectral variation across two XRGs, 3C 223.1 and 3C 403. Although a careful analysis of photometric and spectroscopic evidence led Dennett-Thorpe et al. (2002) to conclude there is no evidence in the galaxy or its environs for a recent merger activity in either 3C 223.1 or 3C 403 (see, also, Landt et al. 2010, for a similar assertion about a larger sample of XRGs), they point out that the backflow models are not consistent with the relative sizes and spectra of the wings in these sources. Hence Dennett-Thorpe et al. suggest that the required rapid change in the jet axis within several Myr is either caused by a delayed SMBH merger following ingestion of a small galaxy by the radio galaxy, which left no obvious signature in the latter's stellar population, or, alternatively, the axis flip may have occurred due to accretion disk instabilities (e.g., Natarajan \& Pringle 1998).

In summary, the basic difference between the spin-flip and the backflow diversion models is that in the latter case both the primary and secondary lobe pairs form and evolve quasi-simultaneously. However, in the spin-flip scenario only the secondary lobe pair (presently appearing as the wings) existed prior to the SMBH spin-flip episode and the currently active primary lobe pair was created after the spinflip.

While discussing the three main approaches to model the XRG phenomenon, we have tried to underscore the difficulties each of those mechanisms encounters when confronted with the available range of observations. In Sect. 3 we shall consider a new scenario which appears to surmount the problems inherent to the existing models. Later, in Sect. 4 we shall contrast all four XRG models in a condensed tabular presentation.

\section{A JET-SHELL INTERACTION MODEL FOR XRGS}

While this proposed new mechanism for XRG formation assumes that a galaxy merger has occurred, it does not necessarily require a spin-flip of the central engine, in that the entire observed radio structure may have formed only after the merger is essentially complete. Originally mooted in GBW03, this mechanism is premised on an interaction between the radio galaxy jets and stellar shells (e.g., GopalKrishna \& Chitre 1983) of the kind that are detected around $\sim 10 \%$ of nearby early-type galaxies located in low density environments (Malin \& Carter 1983; Pierfederici \& Rampazzo 2004; Sikkema et al. 2007). The shells are now also known to be fairly rich in gas (see below). Striking examples of jet-shell interactions on kiloparsec scale have been noticed in the nearest radio galaxy, Centaurus A, in particular, the abrupt flaring of the Northern inner jet right at the point of its impact on a shell (GopalKrishna \& Saripalli 1984, hereafter GS84; Gopal-Krishna \& Wiita 2010, hereafter GW10). In these papers it was also suggested that the S-shaped symmetry of the pattern of radio peaks about the galaxy is indicative a general clockwise rotation of the shell complex. A similar scenario, involving rotating 
shells is proposed here for XRGs and can explain the observed oppositely directed geometry of the wings. We have argued that such interactions in Cen A may be responsible for the production of many of the detected ultra-high energy cosmic rays (Gopal-Krishna et al. 2010). In several nearby ellipticals radial sequences of such shells straddling the galaxy have been detected (e.g., Malin \& Carter 1980, 1983; Malin, Quinn \& Graham 1983; Prieur 1988; Sikkema et al. 2007). An elegant interpretation for these shells in terms of a phase-wrapping formalism has been put forward, which invokes a merger of a disk galaxy with a massive elliptical (Quinn 1984; Dupraz \& Combes 1986; Hernquist \& Quinn 1988).

Clear signatures of such a merger indeed exist in the case of Cen A (see Israel 1998, for a review). Since substantial quantities of $\mathrm{HI}$ and molecular gas have since been detected in the Cen A shells (Schiminovich et al. 1994; Charmandaris et al. 2000; Oosterloo \& Morganti 2005), this very close-by system provides a unique laboratory for studying the jet-shell interaction process. There, at two locations, the northern radio jet is seen being interrupted by a gaseous shell and in each of those encounters the jet is found to bend and flare-up towards the same (north-eastern) side, giving rise to the two local radio peaks called the "northern inner lobe" and the "northern middle lobe" (GW10; GS84). As argued in these papers, such repeated interruptions of the Cen A jets by the rotating gaseous shells at different distances from the nucleus can explain the observed multi-peaked, S-shaped, morphology of its radio lobes. Here we argue that such directly observed jet-shell interactions might hold vital clues for understanding the XRG phenomenon as well. Shells have also been detected in the XRGs 3C 403 and 4C +00.58 (Almeida et al. 2011; Hodges-Kluck, et al. 2010b). Unfortunately, since powerful radio galaxies are rare in the local universe, all XRGs are sufficiently far away so that the vast majority of shells that they may possess cannot be detected with current instrumentation. However, given the frequency of their detection around nearby elliptical galaxies that host RGs (e.g., Sikkema et al. 2007), it is most reasonable to investigate the hypothesis that they are present in many such host galaxies and that suitably oriented jets will interact with them as a matter of course.

Since the relativistic plasma jets in XRGs are typically $1-2$ orders of magnitude more powerful than their counterparts in Cen A, they are unlikely to be totally disrupted upon encountering the shells. It is much more plausible that such encounters would slow down the jet and the lateral kick imparted by the rotating shell would bend it sideways fairly abruptly in a fashion somewhat similar to the situation seen in Cen A, as mentioned above. It is important to note that gas and dust have been detected in shells around several other nearby elliptical galaxies (e.g., Schiminovich et al. 1995; Balcells et al. 2001; Sikkema et al. 2007), while some cool gas has been detected in a very large number of radio-AGN hosts (e.g., Sadler \& Gerhard 1985; Oosterloo et al. 2002) although it cannot be resolved sufficiently to indicate that much of it is associated with shells. Thus it is quite reasonable to expect that more distant elliptical galaxies, hosting more powerful radio jets, also possess gaseous shells which, however, cannot be detected with current instrumentation.

An additional impetus for our pursuing the jet-shell collision scenario for XRGs comes from the clear detection of a jet-cloud collision in the radio galaxy 3C 321 at a redshift $z=0.096$ (Fig. 1 of Evans et al. 2008). It is seen that the western jet of this powerful RG, while being impacted by the ISM of a northward moving gas-rich galaxy, has undergone a sharp bend (by $\sim 40^{\circ}$ ). Both this deflected jet plume, which lacks a terminal hot spot, and the collimated main jet which has, post-interruption, resumed its original course and terminates in a radio/X-ray hot spot, are presently visible in this source. Evans et al. have estimated that the jet's interruption by the moving galaxy lasted for $<2 \times 10^{6} \mathrm{yr}$. Thus, within the XRG terminology, the western half of this RG exhibits both an active (primary) radio lobe and a relic secondary lobe (wing), closely resembling the XRG type radio structure.

Several powerful radio-loud quasars have long been known to show evidence for abrupt bends on large scales, due to which they have been named "dog-leg" quasars and interactions with dense clouds or even nearby galaxies have been suggested to explain their morphology (Stocke, Burns \& Christiansen 1985). We note that evidence for jet-cloud interactions on smaller scales is also available, e.g., the cases of the nearby Seyfert I galaxy III Zw 2 (Brunthaler et al. 2005) and of the Broad Absorption Line quasar, QSO 1045+352 (Kunert-Bajraszewska et al. 2010).

In this context, we stress that the key requirement of a large temporary deflection of a light jet colliding with a few times wider massive cloud, along with subsequent stable flow, has been shown to be 
possible by multiple groups performing 3D numerical simulations with different well tested hydrodynamics codes (de Gouveia Dal Pino 1999; Higgins, O’Brien \& Dunlop 1999; Wang, Wiita \& Hooda 2000). Detailed simulations of light jets passing through many small clouds show significant deviations to the jet flow, though not the large bends that can be achieved by striking large clouds (Sutherland \& Bicknell 2007). 3D simulations of relativistic jets striking massive clouds also show structures similar to that seen in 3C 321 forming under suitable circumstances (Choi, Wiita \& Ryu 2007) and lasting for significant times. The remarkable observational finding in 3C321, together with the two clear incidences of jet/shell collisions observed in Cen A (see above), taken together with the results of the 3D simulations, suggest the following physically motivated scenario for the formation of XRGs.

Usually, multiple shells are prominently observed within a bi-conical region centered at the elliptical galaxy and having its axis broadly aligned with the optical major axis of the elliptical (Malin et al. 1983), a result that is consistent with computations of phase wrapping following a merger (Quinn 1984). The existence of shells on opposite sides of the elliptical, forming a bi-conical pattern broadly aligned with the elliptical's major axis, is particularly conducive for the success of XRG formation scenario based on jet-shell interaction. Such 'aligned' systems of shells are preferentially found in elongated ellipticals (Prieur 1990 and references therein). Accordingly, only the radio jets emerging along the optical major axis would suffer significant interruptions by the shells. The resulting lateral impact on the jets, if adequate in strength and duration, would deflect them, giving rise to a pair of radio wings typically extruding at a large angle from the jet's original direction, as observed in 3C 321 (see above).

This observationally inspired scenario is capable of explaining the two main trends established for XRGs, namely, the strong tendency for their wings to be aligned with the optical minor axis of the host elliptical and for the primary lobe axis to be roughly along the optical major axis (e.g., C02; SS09; HK10a). Simultaneously, it yields a simple explanation for the seemingly puzzling result that the wings are often comparable, or even larger than the primary lobes (Sect. 2.2), which is an embarrassment for the backflow deflection model for XRGs (see below). As discussed in Sect. 2.2, Capetti et al. (2002), in an attempt to address this formidable problem, have suggested that the driving mechanism for the backflow diversion is over-pressure of the radio cocoon, out of which a loosely collimated outflow of the synchrotron plasma would ensue along a direction dictated by the buoyancy forces. Conceivably, the wings forming in this manner can grow (along the optical minor axis) as rapidly as the primary lobes (Sect. 2.2). However, as noted above, it is unclear if the radio lobes are in fact over-pressured (e.g., HK10a). Moreover, as noted by these authors, in the well known XRG NGC 326, the ISM cannot be the 'confining medium' required for the hypothesized over-pressured cocoon, since the northern secondary lobe originates well outside the ISM, although if there is confinement by hot gas associated with a group or cluster then this gas might provide adequate confinement. Recall that in this XRG the secondary lobes again are a few times longer than the primary lobes (Worrall et al. 1995; Murgia et al. 2001).

We wish to stress that, in this new model, if a post-merger RG has a jet orientation independent of the optical axis, as seems to be the case for powerful sources (Battye \& Browne 2009), then only the random fraction of jets launched along the major axes are likely to encounter shells and thereby possibly form XRGs through this mechanism. The majority of jets would not end up being intercepted by the shells and would hence evolve as normal RGs, as is of course observed, since XRGs are a small fraction of all RGs. If the preference for jets to roughly align with the optical minor axis, as seen in weaker RGs, is at all relevant for XRGs, whose powers tend to be near the borderline between the FR I and FR II sources, then this preference for the minor axis would imply that fraction of sources actually aimed toward the major axes would be less than expected from purely random jet/galaxy orientations. Still, we can speculate about a mechanism that would produce an observed correlation between the jet direction and the galaxy's optical major axis, at least as seen in projection. If the incoming, smaller galaxy has no central SMBH, as appears to be the case with M33 and other dwarf galaxies (e.g., Ferrarese et al. 2006), then during the merger the spin of the SMBH in the primary galaxy would not be altered, nor would the jet direction reoriented. Nonetheless, if one averages over all possible directions, the random direction of a jet is expected to be within 30 degrees of any plane. Of course as we can only see the entire configuration of the merged galaxies in projection, it therefore would not be surprising if the jet hits a shell. 


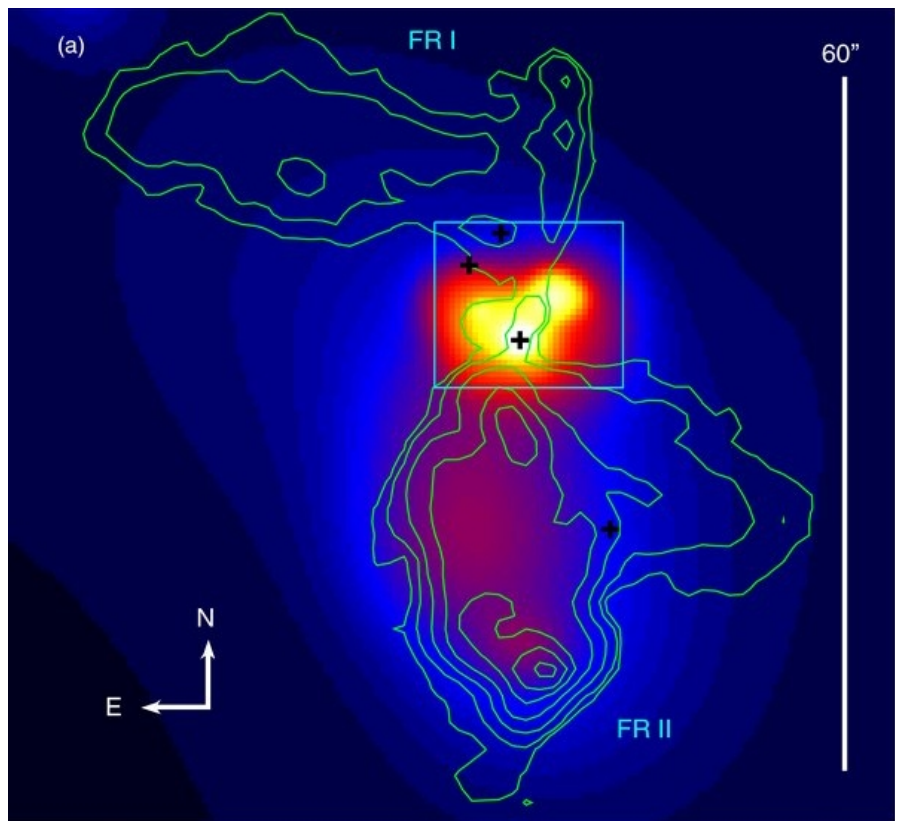

Fig. 1 Radio contours overlain on X-ray emission for the XRG 3C 433. Reprinted by permission from Miller \& Brandt (2009); copyright AAS

As noted in Biermann \& Kronberg (1983) the extent of the outer fringes of the ISM in early Hubble type galaxies can be quite large; however, the pressure in the ISM strongly decreases outward and the pressure in intra-group medium or even intra-cluster medium is very low. The observed near-orthogonal deflection of the two jets in opposite directions, giving rise to the pair of secondary lobes, should occur naturally in the jet-shell encounter model proposed here. Moreover, in this model the size of the wings is determined not mainly by the speed of the backflow within the radio lobes (vis-à-vis the speed of the hot spots). Instead, it would be determined by the net duration over which the jet remained interrupted by the shell(s), as compared to the phase(s) during which the jet advanced uninterrupted by the shell(s). In Fig. 1 a striking realization of the scenario proposed here can be seen in the recent VLA image of the RG 3C433 (Miller \& Brandt 2009: see also, Holt et al. 2007; Tadhunter et al. 2000). ¿From the radio contours, the northern jet certainly appears to have resumed its advance recently, after successfully boring its way through a (hypothetical) shell which has caused a prolonged interruption of the jet that has quite plausibly resulted in the radio wing extending to the east. Surviving evidence for the interruption of the jet in the past is seen in the form of the compact radio peak (N3) precisely at the point of the jet/shell interaction, which can be seen in this case thanks to the sub-arc second resolution of the VLA map by Black et al. (1992). Such high quality maps are, unfortunately, not available for most XRGs so the signatures of jet/shell interactions are usually less clear as it is the case for nearby radio galaxies such as $3 \mathrm{C} 433$ and Cen A.

Thus, this simple picture can readily allow for the possibility of secondary lobes (wings) being more extended than even the primary lobes (GW10), as observed in several XRGs (e.g., 3C 223.1 and 3C 403, Dennett-Thorpe et al. 2002; NGC 326, Murgia et al. 2001; J1130+0058, Zhang et al. 2007; 4C+00.58, Hodges-Kluck et al. 2010b). At the same time, it is clear that even if jets' interuptions by the shells (disposed roughly along the optical major axis) are not strong enough to cause significant diversion of their flow, the repeated stalling of the jets would retard their advance. This could well be why giant radio sources are preferentially seen to align with the optical minor axis of the host galaxy, as found by SS09, Palimaka et al. (1979) and Guthrie (1980). Lastly, the observed Z-shaped morphology of the secondary lobe pair (GBW03) about the nucleus is clearly another natural outcome of this scenario. We note that 
this assertion would hold even if the Z-deflections of the two jets occur at vastly different separations from the nucleus, as for instance seen in the XRG NGC 326 (see above). This is because, in this scenario, the deflection points of the two jets are determined by the radial locations of the two (partial) shells, on the opposite sides of the galaxy along its major axis, which were successful in effectively interrupting and deflecting the respective jets.

The jet-shell interaction model proposed here for XRGs may also be invoked for understanding another intriguing recent results about powerful radio galaxies. It has been noted that the lobes of RGs display more pronounced lateral distortions when the radio source axis is better aligned with the major axis of the host elliptical (SS09). This can be readily understood since the interaction of the lobe plasma with the shells that causes the lobes' distortion would happen mostly when the lobes happen to lie along the host's major axis, i.e., where the shells preferrentially form. Finally, the jet-shell collision scenario for the formation of wings may even apply in the exceptionally rare case of the XRG 4C+00.58, where the jet appears aligned with the optical minor axis, provided a gradual jet reorientation via accretion, as inferred by Hodges-Kluck et al. (2010b), is a valid description of the system. Bearing in mind the vast potential of this scenario for explaining several intriguing morphological aspects of double radio sources, we strongly encourage deep optical searches for the shells about the host galaxies of XRGs, whose counterparts in several nearby ellipticals were discovered already three decades ago (Malin \& Carter 1980, 1983; Malin et al. 1983) and whose relevance to the intriguing RG morphologies was recognized soon thereafter (Gopal-Krishna \& Chitre 1983; GS84). In this context, it is encouraging that a rich system of shells has recently been discovered in the archetypal XRG 3C403 (Almeida et al. 2011). Likewise, it is also important to establish the nature of the environment around XRGs; in the jet-shell interaction picture they are expected to reside in comparatively low density environment, like the shell galaxies. It may also be noted that even on parsec scale jets seem to get repeatedly interrupted by gas clouds, as revealed by the extensive VLBI observations of the nearby Seyfert I galaxy III Zw 2 (Brunthaler et al. 2005).

\section{SUMMARY AND MAIN CONCLUSIONS}

In the foregoing sections we have endeavoured to collate various available clues in order to arrive at an improved understanding of the mechanism responsible for the X-shaped radio galaxies (XRGs). Thus, we confronted the various proposed models with a fairly large body of observations available now. Although rather enigmatic, XRGs may not be rare objects, when it is realized that the XRG classification demands not merely that the primary and secondary lobe axes have a large misalignment, but also that they are oriented far from the line of sight. A condensed overview of the principal merits and shortcomings of the various proposed models is presented in Table 1.

While an occasional XRG might arise from the superposition of two independent AGN inside a merging pair of ellipticals this explanation does not accord with the bulk of the data. A simple buoyancy aided back-flow diversion model (e.g., LW84) might be adequate to explain RGs with modest "wings", where the secondary lobes are short compared the primary. The only way this class of models could account for the not-infrequent occurence of secondary lobes comparable or even larger than the primary lobes, is for the backflow filled cocoon to become strongly over-pressured, so that the relativistic plasma might rapidly squirt out and escape along the minor axis of the host galaxy. If then, the primary jets happen to be fortuitously oriented along the host elliptical's major axis, the interesting correlation found by Capetti et al. (2002) can probably be understood. However, the numerical simulations purporting to demonstrate this is feasible were long restricted to two-dimensions (C02), and this artificially induced symmetry is very likely to exaggerate the collimation and strength of these outflows. However, very recent 3-D simulations have demonstrated that it is possible to obtain lengthy overpressured outflows if the host galaxy has a very high ellipticity (Hodges-Kluck et al. 2011); if viewed at particularly favorable orientations the secondary lobes can even appear to be longer than the primary ones in projection (Hodges-Kluck et al. 2011; Wiita et al. 2011).

In addition, the over-pressured cocoon model does not seem capable of explaining the important Z-symmetric subset of XRGs. Moreover, convincing evidence for the requisite over-pressured regions 
within the cocoons is still lacking. There is a strong evidence that mergers are necessary for triggering at least some AGN and several nearby AGN, such as Cen A, are prime examples of an elliptical having rather recently swallowed a gas and dust rich neighbour. These mergers are of great interest also for the generation of gravitational radiation and perhaps prompt electromagnetic signals as well.

The basic spin-flip model also can readily explain why at most only one pair of lobes is ever of the FR II type. In addition, a simple variant of this basic picture is able to produce the Z-shaped distortion clearly witnessed in some of the XRGs, another important merit. Thus it seems likely that at least some XRGs are indeed created in this manner. The spin-flip model also produces very interesting and specific relations between gravitational radiation and electromagnetic wave signatures (e.g., GB09), and their detection would be extremely important and also would provide proof of the relevance of such mergers in AGN. However, this physically well motivated spin-flip model offers no natural explanation for the correlation of the primary lobe pair with the optical major axis of the parent elliptical, and so probably cannot account, by itself, for the majority of XRGs.

Related to the spin-flip scenario we show in the Appendix A that the probability of any specific mass ratio $q$ is close to a flat distribution in $\Delta q / q$; thus mass ratios close to unity should be exceedingly rare. We also address a number of observations which occur for semi-major mergers (defined as having the most likely mass ratios, between 1:30 to 1:3, 1/10 being typical). In Appendix B, we compute the spin evolution of the radio-loud (primary) SMBH as well as the expected intervals between successive semi-major BH mergers. We find that the spin reorientations during the two phases (separated by the time when the declining orbital momentum of the infalling SMBH has become comparable to the spin momentum of the main SMBH) are similar in magnitude, but the rate of spin evolution is about $10^{4}$ times faster in the second phase, resulting in a duration as short as $\sim 3$ years, while the precession timescale is less then a day. If the rapidly rotating jets are relativistic and if they came close to our line of sight, they could produce significant variability at all wavelengths (but mostly detectable in the radio, hard X-ray and gamma-ray) years before the coalescence. Such systems would provide a detectable EM precursor of the impending strong GW emission.

A careful scrutiny of the mechanisms leading to XRGs shows that while each of them might play a dominant role in different XRGs, the mechanisms involving galaxy mergers, which include the spinflip scenario, are likely to have the widest applicability, considering their greater overall compatibility with the observations. More specifically, the various observational constraints in some sources have led us to propose a jet-shell interaction model for XRGs. While this proposed new alternative mechanism also involves a galaxy merger and assumes that each galaxy possesses a SMBH, as does the spin-flip model, it does not require the galaxy to have been radio loud prior to the merger. However, it may be noted that a spin-flip will typically have occurred in the course of merger and the direction of the jets thus triggered will probably be along the axis defined by the angular momentum of the accreted galaxy and therefore also perpendicular to any central dust-lane. The powerful interruptions of jets by moving massive clouds in the environments of the host galaxy, as observed in some nearby RGs, such as Cen A and 3C 321, provide fairly persuasive evidence in favour of the jet-shell interaction scenario being important in a goodly subset of XRGs. Here it is relevant to recall that the stellar shells (which are probably also gaseous) in a bi-conical pattern are preferentially disposed along the optical major axes of the post-merger galaxy. When these features are combined with simulations that show substantial sideways flows from powerful jets striking massive clouds, the wide range of morphological properties associated with XRGs can be understood.

\section{ACKNOWLEDGMENTS}

We thank the referee for pointing out important additional references and for suggestions that clarified the presentation. PLB wishes to thank Lakshmi Saripalli, Chanda Jog and Elisabete de Gouveia Dal Pino for extended discussions and gratefully acknowledges the award of a Sarojini Damodaran Fellowship (2009). Support for work by PLB has come from the AUGER membership and theory grant 05 CU 5PD $1 / 2$ via DESY/BMBF and VIHKOS. LÁG is grateful to Tiberiu Harko for kind hospitality during his visit at the University of Hong Kong. He was partially supported by the Hungarian Scientific Research 
Table 1. Comparison of the salient aspects of the main models of XRGs.

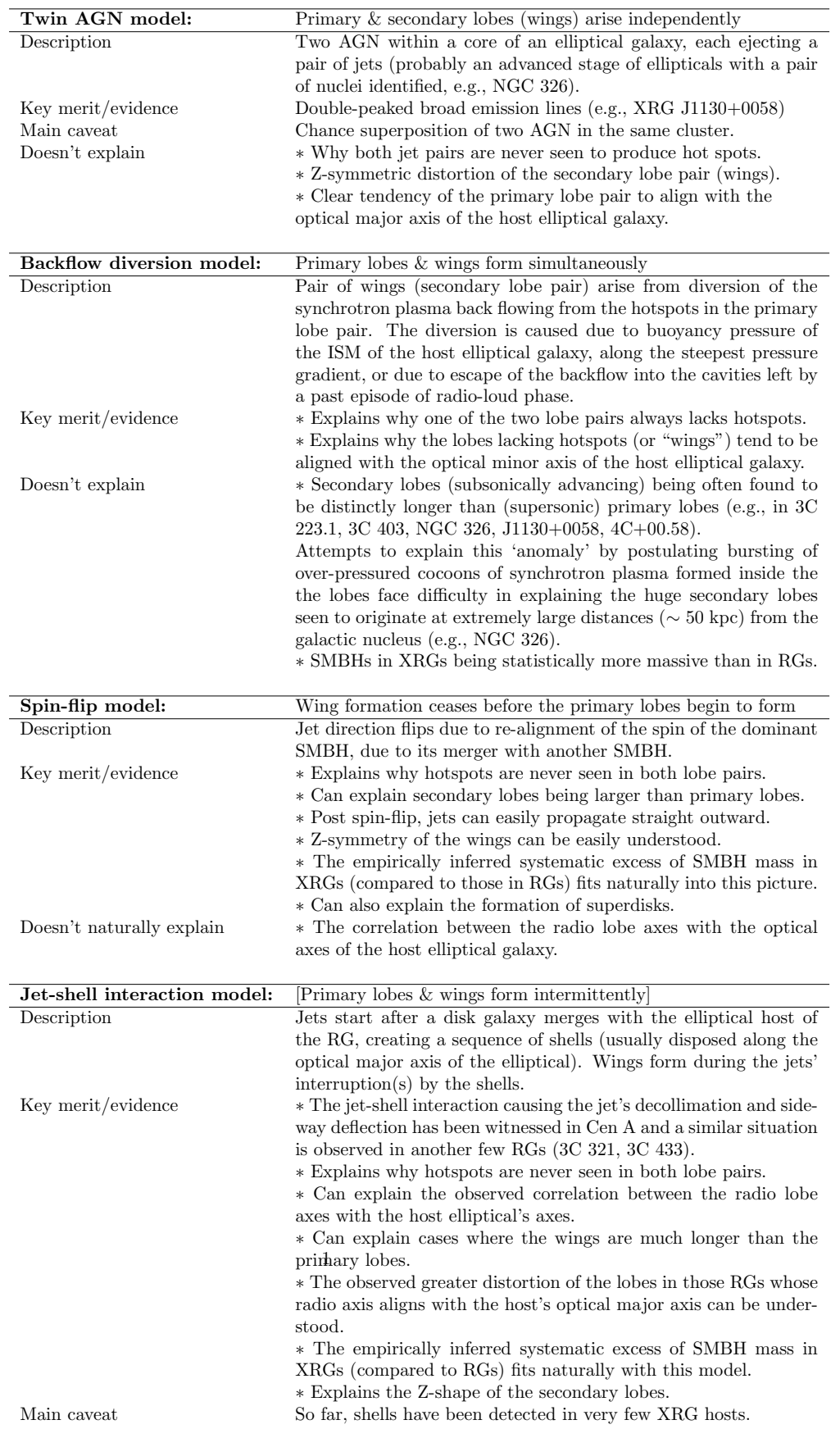

Fund (OTKA) grant no. 69036 and by COST Action MP0905 "Black Holes in a Violent Universe". The work of PJW was supported in part by a subcontract to US NSF grant AST 05-07529 to the University of Washington. 


\section{Appendix A: THE EXPECTED MASS RATIO OF MERGING SMBHS AND CONSEQUENCES}

In GB09 the characteristic merger ratio was estimated to be between 3:1 to 30:1, the value of 10:1 being found typical. Here we give independent considerations supporting the statement that both equal mass encounters and extreme mass ratio mergers, which qualify as a test particle falling into a black hole, are rare.

Mergers between two black holes both drawn from a black hole mass distribution can be written as integrals over a merger rate $\alpha\left(M_{1}, M_{2}\right)$ multiplied by the density at each of the masses $M_{1}$ and $M_{2}$. This approach uses the assumption that the highest density region of the universe are in groups and clusters, where the density depends (actually does not depend) on redshift as $(1+z)^{0}$ (Cavaliere et al. 1991, $1992,1997)$ up to some critical redshift. Silk \& Takahashi (1979) have found an analytic asymptotic solution to the merger equation using a merger rate running as $M_{1}^{\epsilon / 2} M_{2}^{\epsilon / 2}$. We can reproduce the data to a good approximation with a merger rate varying as $M_{1}^{2 / 3} M_{2}^{2 / 3}$; in fact, this exponent, $\epsilon=4 / 3$, is the gravitational focusing limit in the analysis of Silk \& Takahashi. This gives a low mass tail of $M^{-2}$ for the BH mass distribution, and a high mass tail, which can be approximated by $M^{-3}$, quite close what the data show (Caramete $\&$ Biermann 2010). These two regimes are joined at a break mass $M_{b}$ of about $10^{8} M_{\odot}$.

Including mergers between two black holes both below the bend, 60 percent of all mergers occur within the mass ratio ranges 3:1 and 100:1. Ignoring mergers between two partner black holes both below the break mass we work out the frequency in various mass ratio ranges in more detail: for the ratio ranges $q=1$ to 3,3 to 10,10 to 30 , and 30 to 100 , the corresponding percentages are $3,15,61$ and 21 percent. Therefore the most common mass ratio is 10 to 30 with 61 percent of all mergers, and an additional 36 percent of mergers occur in the two adjoining mass ranges. Therefore, the mass ratio range from 3:1 and 100:1 covers 97 percent of all mergers.

Under specific initial conditions the linear momentum kick imparted to the merged SMBHs can exceed several thousand $\mathrm{km} \mathrm{s}^{-1}$, which is enough for the merged SMBH to escape from the host elliptical (Bogdanović, Reynolds \& Miller 2007). These large linear momenta can only be produced when the BH mass ratio is nearly 1 , and for restricted spin configurations (anti-aligned spins, lying in the orbital plane). We will argue below that such initial conditions are unlikely. First, as seen above, the probability of any specific mass ratio $q$ is close to a flat distribution in $\Delta q / q$; thus mass ratios close to unity should be exceedingly rare.

Secondly, any gas/circumbinary disk will act toward an alignment of the spins with the orbital momentum, significantly reducing the kick velocity (Bogdanovic et al. 2007). This occurs through the Bardeen-Petterson effect (Bardeen \& Petterson 1975), which in turn is based on the Lense-Thirring precession. The real situation is expected to fall between the two rare extremes, namely, an extremely 'wet' merger (i.e., copious amount of gas associated with it, leading to this pre-merger realignment), and a completely 'dry' merger with essentially no gas (hence allowing for random spin orientations). Therefore it is expected that even the presence of some gas or a circumbinary disk will disfavour the spin configuration leading to a large recoil, but still leave room for a significant spin-flip.

Consequent to a 'semi-major' merger the SMBH spin could undergo a large directional 'flip'. The new spin axis would tend to get reoriented towards the orbital momentum axis of the in-spiraling massive BH (and, therefore, the ISM rotation axis: see GB09). Such a geometry would ensure, firstly, that the post-merger jets, ejected along the new spin axis, will encounter little lateral impact from the rotating ISM and hence propagate essentially straight outward. Secondly, the old lobes would gradually acquire the appearance of wings/relics which would be kept energized at a significant level as the synchrotron plasma backflowing from the new hot spots would find a natural escape sideways into these pre-existing low-pressure relic lobes (GBW03; LW84).

Following a semi-major merger (SMBH mass ratio $>1: 30$ ) the spin axis of the coalesced SMBH pair is expected to settle down roughly along the direction of the original orbital angular momentum of the secondary galaxy about the primary one (GB09). Then the same axis is likely to be retained 
during any subsequent ejections of restarted jets. This is because the probability of recurring semimajor mergers, each causing a large spin-flip is quite small, in that the temporal interval between them certainly is $>1$ Gyr for semi-major mergers with mass ratios $>1: 30$ and even longer for major mergers (Z. Lippai, private communication, based on the merger tree program in Lippai et al. 2009). Whereas, if jets are restarted while the earlier lobes are still visible, then it is likely that they have done so within $\sim 10^{8} \mathrm{yr}$ of the launch of the original jets, since visible radio activity seldom lasts much longer than that (e.g., Blundell \& Rawlings 1999; Gopal-Krishna \& Wiita 2001; Barai \& Wiita 2007; Kaiser $\&$ Best 2007). In this way, the situations where the incipient inner lobe pair is essentially aligned with the (outer) primary lobes, as shown for a few XRGs (SS09), can be explained naturally. An alternative possibility for such an observed alignment is from a fortuitous projection of a real XRG onto the plane of the sky in such a way that the shorter primary lobes appear embedded within the decaying secondary lobes, thereby giving the appearance of a "double-double" source (Wiita et al. 2011).

\section{Appendix B: THE EXPECTED TIME EVOLUTION OF THE SMBH SPIN}

In GB09 the following evolution of the inclination angle was derived

$$
\dot{\alpha} \approx \frac{32 c^{3}}{5 G m} \varepsilon^{9 / 2} \eta \nu^{-1}\left(\frac{L}{J}\right)^{2} \sin (\alpha+\beta),
$$

with $\varepsilon=G m / c^{2} r$ the post-Newtonian parameter (increasing during the inspiral with the decreasing separation $r$; here $m=m_{1}+m_{2}$ is the total mass), $\alpha$ and $\beta$ the angles spaned by the orbital angular momentum and the spin with the total angular momentum (their sum, $\alpha+\beta$ being constant during the inspiral), $\nu=m_{2} / m_{1} \leq 1$ is the mass ratio, $\eta=m_{1} m_{2} / m^{2}=\nu(1+\nu)^{-2}=\mu / m$, the symmetric mass ratio and $J$ the magnitude of the total angular momentum. For mutually perpendicular spin and orbital angular momentum $J^{2}=L^{2}+S_{1}^{2}$. For circular orbits and to leading order, $S_{1} / L=\varepsilon^{1 / 2} \nu^{-1} \chi_{1}$ holds (where $\chi_{1} \in[0,1]$ is the dimensionless spin), and we now obtain

$$
\left(\frac{L}{J}\right)^{2}=\left(1+\varepsilon \nu^{-2} \chi_{1}^{2}\right)^{-1}
$$

thus the evolution of the inclination angle is described by

$$
\dot{\alpha} \approx C \nu^{-1} \varepsilon^{9 / 2}\left(1+\varepsilon \nu^{-2} \chi_{1}^{2}\right)^{-1},
$$

with the constant coefficient given as

$$
C=\frac{32 c^{3}}{5 G m} \eta \sin (\alpha+\beta)
$$

We note that $\dot{\alpha}$ as a function of $\varepsilon$ is monotonically increasing, thus the spin-flip rate increases with increasing post-Newtonian parameter (with decreasing radius).

In order to find $\delta \alpha$ during any part of the inspiral we have to integrate

$$
\delta \alpha=\int \dot{\alpha}(r) d t=\int \frac{\dot{\alpha}(r)}{\dot{r}_{g w}} d r,
$$

where by $\dot{r}_{g w}$ we mean the rate of the inspiral. As for a Keplerian circular orbit $L^{2}=G m \mu^{2} r$, therefore $\dot{r}_{g w}=2\left(G m \mu^{2}\right)^{-1} L \dot{L}^{g w}$. Employing

$$
\dot{L}^{g w}=-\frac{32 G \mu^{2}}{5 r}\left(\frac{G m}{c^{2} r}\right)^{5 / 2},
$$


which holds on the radial orbit in average (Apostolatos et al. 1994), we get

$$
\dot{r}_{g w}=-\frac{64 \eta}{5} c \varepsilon^{3}
$$

thus

$$
\delta \alpha \approx \frac{5 C}{64 \eta \nu c} \int \frac{\varepsilon^{-1 / 2}}{1+\varepsilon \nu^{-2} \chi_{1}^{2}} d \varepsilon .
$$

For mass ratios about $\nu \approx 0.1$ the inspiral begins at about $\varepsilon^{*} \approx 10^{-3}$ (GB09), it proceeds through the $L \gg S_{1}$ phase until $L \approx S_{1}$ is reached (when $\varepsilon \approx \nu^{2}$ ), then continues through the phase $L \ll S_{1}$ until $\varepsilon_{f i n}=\left(G m / c^{2} r_{m s}\right)$, where $r_{m s}$ is the marginally stable orbit. For high spin $\left(\chi_{1} \sim 1\right)$ we have $r_{m s} \sim 2 G m / c^{2}$ thus $\varepsilon_{f i n} \sim 0.5$. Let us estimate the ratio of the changes in the inclination angle (the ratio of spin-flips) during these two phases. We obtain

$$
\frac{\delta \alpha_{I}}{\delta \alpha_{I I}}=\frac{\int_{10^{-3}}^{10^{-2}} \frac{\varepsilon^{-1 / 2}}{1+100 \varepsilon} d \varepsilon}{\int_{10^{-2}}^{0.5} \frac{\varepsilon^{-1 / 2}}{1+100 \varepsilon} d \varepsilon}=0.74
$$

Therefore, the respective spin-flips are comparable.

Now let us see how long the phases last. For this we evaluate in a similar way

$$
\delta t=\int_{t_{1}}^{t_{2}} d t=\int_{r_{1}}^{r_{2}}\left|\frac{d r}{\dot{r}_{g w}}\right|=-\frac{5 G m}{64 \eta c^{3}} \int_{\varepsilon_{1}}^{\varepsilon_{2}} \varepsilon^{-5} d \varepsilon=\left.\frac{5 G m}{256 \eta c^{3}} \varepsilon^{-4}\right|_{\varepsilon_{1}} ^{\varepsilon_{2}},
$$

to see that the time-scale varies linearly with the total mass. So then we have

$$
\frac{\delta t_{I}}{\delta t_{I I}}=\frac{\int_{10^{-3}}^{10^{-2}} \varepsilon^{-5} d \varepsilon}{\int_{10^{-2}}^{0.5} \varepsilon^{-5} d \varepsilon} \approx 9999 .
$$

We conclude that the second part of the spin-flip occurs approximately $10^{4}$ times faster than the first part. Due to the increased post-Newtonian parameter and increased rate of change of the inclination angle, a considerable and certainly observable part of the spin-flip will occur during the last stages of the inspiral. An estimate for this was given in GB09 as 3 years.

\section{References}

Almeida, C.R., Tadhunter, C.N., Inskip, K.J, Morganti, R., Holt, J., \& Dicken, D. 2011. MNRAS, 410, 1550

Apostolatos, T.A., Cutler, C., Sussman, G.J., \& Thorne, K.S. 1994, Phys. Rev. D, 49, 6274

Balcells, M., van Gorkom, J.H., Sancisi, R., \& del Burgo, C. 2001, AJ, 122, 1758; Erratum: 2001, AJ, 122,3508

Barai, P. \& Wiita, P.J. 2007, ApJ, 658, 217

Bardeen, J.M., \& Petterson, J. A. 1975, ApJ, 195, L65

Battistini, P., Bonoli, F., Silvestro, S., Fanti, R., Gioia, I.M., \& Giovannini, G. 1980, A\&A,, 85, 101

Battye, R.A., \& Browne, I.W.A. 2009, MNRAS, 399, 1888

Becker, R.H., White, R.L., \& Helfand, D.J. 1995, ApJ, 450, 559

Begelman, M.C., Blandford, R.D., \& Rees, M.J. 1980, Nature, 287, 307

Begelman, M.C., Blandford, R.D., \& Rees, M.J. 1984,Rev. Mod. Phys., 56, 225

Benson, A.J., Dzanović, D., Frenk, C.S., \& Sharples, R. 2007,MNRAS, 379, 841

Bicknell, G.V. 1994, ApJ, 422, 542

Bicknell, G.V. 1995,ApJS, 101, 29

Biermann, P.L., \& Kronberg, P.P. 1983, ApJ, 268, L69

Biermann, P.L., et al. 2005, in Proc. Paris Conference on Cosmology, June 2000, ed. N. Sanchez, H. de Vega (Paris: IAP), 148; astro-ph/0211503 
Black, A.R.S., Baum, S.A., Leahy, J.P., Perley, R.A., Riley, J.M., \& Scheuer, P.A.G. 1992, MNRAS, 256, 186

Blandford, R.D., \& Rees, M.J. 1974, MNRAS, 169, 395

Blundell, K.M., \& Rawlings, S. 1999, Nature, 399, 330

Bogdanović, T., Reynolds, C. S., \& Miller, M. C. 2007, ApJ, 661, L147

Boroson, T.A., \& Lauer, T.R. 2009, Nature, 458, 53

Brunthaler, A., Falcke, H.. Bower, G. C., Aller, M. F., Aller, H. D., \& Teräsranta, H. 2005, A\&A, 435, 497

Camenzind, M. 1987, A\&A, 184, 341

Capetti, A., Zamfir, S., Rossi, P., Bodo, G., Zanni, C., \& Massaglia, S., 2002. A\&A, 394, 39 (C02)

Capetti, A., Raiteri, C.M., \& Buttiglione, S. 2010, A\&A, 516, 59

Caramete, L.I., \& Biermann, P.L. 2010, A\&A, 521, 55

Cavaliere, A., \& Menci, N. 1997, ApJ, 480, 132

Cavaliere, A., Colafrancesco, S., \& Scaramella, R. 1991, ApJ, 380, 15

Cavaliere, A., Colafrancesco, S., \& Menci, N. 1992, ApJ, 392, 41

Charmandaris, V., Combes, F., \& van der Hulst, J.M. 2000, A\&A, 356, L1

Cheung, C.C. 2007, AJ, 133, 2097 (C07)

Cheung, C.C., et al. 2009, ApJS, 181, 548

Choi, E., Wiita, P.J., \& Ryu, D. 2007, ApJ, 655, 769

de Gouveia Dal Pino, E.M. 1999, ApJ, 526, 862

Dennett-Thorpe, J., et al. 2002, MNRAS, 330, 609

Donea, A.C., \& Biermann, P.L. 1996, A\&A, 316, 43

Dotti, M., et al. 2006, MNRAS, 372, 869

Dupraz, C., \& Combes, F. 1986, A\&A, 166, 53

Ekers, R.D., Fanti, R., Lari, C., \& Parma, P, 1978, Nature, 276, 588

Evans, A.S., Sanders, D.B., Surace, J.A.,\& Mazzarella, J.M. 1999, ApJ, 511, 730

Evans, D.A., et al. 2008, ApJ, 675, 1057

Faber, S.M., et al. 1997, AJ 114, 1771

Falceta-Gonçalves, D., Caproni, A., Abraham, Z., Teixeira, D.M., \& de Gouveia Dal Pino, E.M. 2010, ApJ, 713, L74

Falle, S.A.E.G. 1991, MNRAS, 250, 581

Fanaroff, B.L., \& Riley, J.M. 1974, MNRAS, 167, 31P

Gergely, L.À., \& Biermann, P.L. 2009, ApJ, 697, 1621

Giovannini, G., et al. 2005, ApJ, 618, 635

Gopal-Krishna. 1991, A\&A, 248, 415

Gopal-Krishna, Biermann, P.L., \& Wiita, P.J. 2003, ApJ, 594, L103

Gopal-Krishna, Biermann, P.L., \& Wiita, P.J. 2004, ApJ, 603, L9

Gopal-Krishna, Biermann, P.L., de Souza, V., \& Wiita, P.J. 2010, ApJL, 720, L155

Gopal-Krishna, \& Chitre, S.M. 1983, Nature, 303, 217

Gopal-Krishna, \& Saripalli, L. 1984, A\&A, 141, 61

Gopal-Krishna, \& Wiita, P.J. 1988, Nature, 333, 49

Gopal-Krishna, \& Wiita, P.J. 1991, A\&A, 248, 415

Gopal-Krishna, \& Wiita, P.J. 2000, ApJ, 529, 189

Gopal-Krishna, \& Wiita, P.J. 2001, ApJ, 560, L115

Gopal-Krishna, \& Wiita, P.J. 2010, NewA 15, 96 (GW10)

Guthrie, B.N.G. 1980, Ap\&SS 70, 211

Heinz, S., et al. 2008, in Extragalactic Jets: Theory and Observation from Radio to Gamma-Ray. ASP

Conf. Ser., Vol. 386, ed. T.A. Rector \& D.S. De Young (San Francisco: ASP), 327

Hernquist, L., \& Quinn, P.J. 1988, ApJ, 331, 682

Higgins, S.W., O’Brien, T.J., \& Dunlop, J.S. 1999, MNRAS, 309, 273

Hodges-Kluck, E., Reynolds, C.S. 2011, ApJ, 733, 58

Hodges-Kluck, E., Reynolds, C.S., Cheung, C.C., \& Miller, M.C. 2010a, ApJ, 710, 1205 
Hodges-Kluck, E., Reynolds, C.S., Miller, M.C., \& Cheung, C.C. 2010b, ApJ, 717, L37

Högbom, J.A., \& Carlsson, I. 1974, A\&A, 34, 341

Holt, J., et al. 2007, MNRAS, ,381, 611

Hooda, J.S., \& Wiita, P.J. 1996, ApJ, 470, 211

Hooda, J.S., \& Wiita, P.J. 1998, ApJ, 493, 81

Hota, A., et al. 2009, in The Low Frequency Radio Universe, ASP Conf. Ser. 407, eds. D.J. Saikia, D.A.

Green, Y. Gupta, \& T. Venturi (San Francisco: ASP), 104

Israel, F.P. 1998, A\&A Rev, 8, 237

Kaiser, C.R., \& Alexander, P. 1997, MNRAS, 286, 215

Kaiser, C.R., \& Best, P.N. 2007, MNRAS, 381, 1548; Erratum: 2008 MNRAS, 384, 1742.

Kawakatu, N. 2009, ApJ, 697, L173

Klein, U., Mack, K.-H., Gregorini, L., \& Parma, P. 1995, A\&A, 303, 427

Kocsis, B., \& Loeb, A. 2008, PRL, 101, id. 041101.

Komossa, S., Burwitz, V., Hasinger, G., Predehl, P., Kaastra, J.S., \& Ikebe, Y. 2003, 582, L15

Komossa, S., Zhou, H., \& Lu, H. 2008, ApJ, 678, L81

Kompaneets, A.S. 1960, Soviet Physics Doklady 5, 46

Konar, C., Saikia, D.J., Jamrozy, M., \& Machalski, J. 2006, MNRAS, 372, 693

Kormendy, J., \& Richstone, D. 1995, ARA\&A, 33, 581

Kraft, R.P., Hardcastle, M.J., Worrall, D.M., \& Murray, S.S. 2005, ApJ, 622, 149

Kunert-Bajraszewska, M., Janiuk, A., Gawroński, M.P., \& Siemiginowska, A. 2010, ApJ, 718, 1345

Kunert-Bajraszewska, M., \& Janiuk, A. 2011, ApJ, 736, 125

Lal, D.V., \& Rao, A.P. 2007, MNRAS, 374, 1085

Landt, H., Cheung, C.C., \& Healey, S.E. 2010, MNRAS, 408, 1103

Lara, L., et al. 1999, A\&A, 348, 699

Lauer, T.R., et al. 2007, ApJ, 662, 808

Leahy, J.P., Muxlow, T.W.B., \& Stephens, P.W. 1989, MNRAS, 239, 401

Leahy, J.P., \& Parma, P. 1992, in Extragalactic Radio Sources: From Beams to Jets, eds. J. Roland, H. Sol, \& G. Pelletier (Cambridge: Cambridge Univ. Press), 307

Leahy, J.P. \& Williams, A.G. 1984, MNRAS, 210, 929

Lippai, Z., Frei, Z., \& Haiman, Z. 2009, ApJ, 701, 360

Liu, F.K. 2004, MNRAS, 347, 1357

Liu, F.K., \& Chen, X. 2007, ApJ, 671, 1272

Liu, F.K., Wu, X.-B., \& Cao, S.L. 2003, MNRAS, 340, 411

Liu, X., Shen, Y., \& Strauss, M.A. 2011, ApJ, 736, L7

Magorrian, J. et al. 1998,AJ, 115, 2285

Malin, D.F., \& Carter, D. 1980, Nature, 285, 643

Malin, D.F., \& Carter, D. 1983, ApJ, 274, 534

Malin, D.F., Quinn, P.J., \& Graham, J.A. 1983, ApJ, 272, L5

Merritt, D., \& Ekers, R.D. 2002, Science, 297, 1310

Mezcua, M., Lobanov, A.P., Chavushyan, V.H., \& León-Tavares, J. 2010, A\&A, 572, 38.

Miller, B.P., \& Brandt, W.N. 2009, ApJ, 695, 755

Morganti, R., Emonts, B., \& Oosterloo, T. 2009, A\&A, 496, L9

Mösta, P., et al. 2010, PRD, 81, id. 064017.

Murgia, M., et al. 2001, A\&A, 380, 102

Nagar, N.M., Falcke, H., \& Wilson, A.S. 2005, A\&A, 435, 521

Nagar, N.M., Wilson, A.S., \& Falcke, H. 2001, ApJ, 559, L87

Nakamura, M., Tregillis, I.L., Li, H., \& Li, S. 2008, ApJ, 686, 843

Natarajan, P., \& Pringle, J.E. 1998, ApJ, 506, L97

Noel-Storr, J., Baum, S.A., Verdoes Kleijn, G., van der Marel, R.P., O’Dea, C.P., de Zeeuw, P. T., \& Carollo, C.M. 2003, ApJS, 148, 419

Oosterloo, T.A., \& Morganti, R. 2005, A\&A, 429, 469

Oosterloo, T.A., Morganti, R., Sadler, E.M., Vergani, D., \& Caldwell, N. 2002, AJ, 123, 729 
Palimaka, J.J., Bridle, A.H., Fomalont, E.B., \& Brandie, G.W. 1979, ApJ, 231, L7

Perez-Fournon, I., \& Biermann, P. 1984, A\&A, 130, L13

Pierfederici, F., \& Rampazzo, R. 2004, Astron. Nachr., 325, 359

Press, W.H., \& Schechter, P. 1974, ApJ, 187, 425

Prieur, J.-L. 1988, ApJ, 326, 596

Prieur, J.-L. 1990, in Dyanmics and Interactions of Galaxies, ed. R. Wielen (Berlin: Springer-Verlag), 72

Quinn, P.J. 1984, ApJ, 279, 596

Rees, M.J. 1978, Nature, 275, 516

Rodriguez, C., Taylor, G.B., Zavala, R.T., Peck, A.B., Pollack, L.K., \& Romani, R.W. 2006, ApJ, 646, 49

Rottmann, H. 2001, PhD thesis, Univ. Bonn; http://hss.ulb.uni-bonn.de/2001/0074/0074.htm

Sadler, E.M., \& Gerhard, O.E. 1985, MNRAS, 214, 177

Sansom, A.E., et al. 1987, MNRAS, 229, 15

Saripalli, L., \& Subrahmanyan, R. 2009, ApJ, 695, 156 (SS09)

Saripalli, L., Subrahmanyan, R., \& Udaya Shankar, N. 2002, ApJ, 565, 256

Schiminovich, D., van Gorkom, J.H., van der Hulst, J.M., \& Kasow, S. 1994, ApJ, 423, L101

Schiminovich, D., van Gorkom, J.H., van der Hulst, J.M., \& Malin, D.F. 1995, ApJ, 444, L77

Schoenmakers, A.P., et al. 2000, MNRAS, 315, 371

Shields, G.A., et al. 2009, ApJ, 707, 936

Sikkema, G., et al. 2007, A\&A, 467, 1011

Silk, J., \& Takahashi, T. 1979, ApJ, 229, 242

Sillanpää, A., et al. 1988, ApJ, 325, 628

Sutherland, R.S. \& Bicknell, G.V. 2007, ApJ Supp. Ser., 173, 37

Burke-Spolaor, S. 2011, MNRAS, 410, 2113.

Tadhunter, C.N., Villar-Martin, M., Morganti, R., Bland-Hawthorn, J., \& Axon, D. 2000, MNRAS, 314, 849

Takahashi, M., Nitta, S., Tatematsu, Y., \& Tomimatsu, A. 1990, ApJ, 363, 206

Valtonen, M. J., et al. 2008, Nature 452, 851

Wang, Y., \& Biermann, P.L. 1998, A\&A, 334, 87

Wang, Z., Wiita, P.J., \& Hooda, J.S. 2000, ApJ, 534, 201

Wiita, P.J., Sobczak, R. \& Starr, J. 2011, in preparation

Wilson, A.S. \& Colbert, E.J.M. 1995, ApJ, 438, 62

Wirth, A., Smarr, L., \& Gallagher, J.S. 1982, AJ, 87, 602

Worrall, D.M., Birkinshaw, M.,\& Cameron, R.A. 1995, ApJ, 449, 93

Zeldovich, Ya., \& Raizer, Yu. 1966, Physics of Shock-Waves and High Temperature Hydrodynamic

Phenomena, 2 Vols., edited by W.D. Hayes \& R.F. Probstein (New York: Academic Press)

Zhang, X.-G., Dultzin-Hacyan, D., \& Wang, T.-G. 2007, MNRAS, 377, 1215

Zier, Ch., \& Biermann, P.L. 2001, A\&A, 377, 23

Zier, Ch., \& Biermann, P.L. 2002, A\&A, 396, 91 